Artigo Original

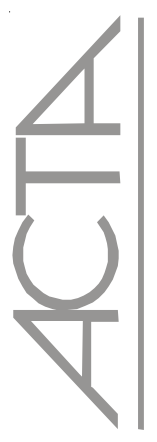

\title{
Percepção do estresse entre enfermeiros que atuam em Terapia Intensiva
}

\author{
Perception of Stress Among Critical Care Nurses \\ Percepción del estrés entre enfermeros que actúan en Cuidados Intensivos

\section{Maria Verônica Guilherme Ferrareze ${ }^{1}$, Viviane Ferreira ${ }^{2}$, Ana Maria Pimenta Carvalho ${ }^{3}$}

\section{RESUMO}

\begin{abstract}
Objetivo: o estudo investigou a ocorrência de estresse entre enfermeiros que atuam na assistência a pacientes críticos de uma Unidade de Cuidados Intensivos em um hospital universitário. Métodos: utilizou-se um instrumento para descrição sociodemográfica da amostra e um inventário para identificar o estresse e a fase em que se encontra. Resultados: a amostra foi composta por 12 enfermeiros, $91,7 \%$ do sexo feminino; 75\% com carga horária de trabalho entre 10-12 horas diárias; 33,4\% com tempo de formação universitária entre 5 e 10 anos. Discussão: mais da metade dos trabalhadores (66,7\%), que assiste pacientes críticos, mostrou sinais de sofrimento físico e/ou psicológico característicos da fase de resistência ao estresse. Conclusão: tais achados sugerem a necessidade de atenção a esses profissionais para que seus sintomas não evoluam para a fase de exaustão.

Descritores: Estresse psicológico; Enfermeiras/psicologia; Enfermeiros/psicologia; Unidades de terapia intensiva; Ambientes de instituições de saúde
\end{abstract}

\begin{abstract}
Objective: to examine the perception of stress among critical care nurses in critical from a Brazilian university hospital. Methods: a demographic questionnaire was used to gather the sample characteristics data. Perception and level of stress was measured with a known stress inventory. Results: the sample consisted of 12 nurses. The majority of the sample was women (91.7\%) women; working 10 to 12 hours per day (75\%), and less than a half of them (33.4\%) had 5 to 10 years of higher education. More than a half of the sample (66.7\%) had signs of physical and/or psychological suffering, which are characteristic of the stress resistance phase. Conclusion: these findings suggest that critical care nurses may need the special attention to prevent the development of stress and its complications.
\end{abstract}

Keywords: Psychological Stress; Nurses; Critical Care Unit; Environment of health care institutions.

\section{RESUMEN}

Objetivo: el estudio investigó la ocurrencia de estrés entre enfermeros que actúan en la asistencia a pacientes críticos de una Unidad de Cuidados Intensivos en un hospital universitario. Métodos: fue utilizado un instrumento para la descripción sociodemográfica de la muestra y un inventario para identificar el estrés y la fase en que se encuentra. Resultados: la muestra estuvo constituida por 12 enfermeros, $91,7 \%$ del sexo femenino; $75 \%$ con carga horaria de trabajo entre 10 a 12 horas diarias; 33,4\% con tiempo de formación universitaria entre 5 y 10 años. Discusión: más de la mitad de los trabajadores (66,7\%), que asiste a pacientes críticos, mostró señales de sufrimiento físico y/o psicológico característicos de la fase de resistencia al estrés. Conclusión: tales hallazgos sugieren la necesidad de atención a esos profesionales para que sus síntomas no evolucionen hacia la fase de agotamiento.

Descriptores: Estrés psicológico; Enfermeras/psicología; Enfermeros/psicología; Unidades de cuidados intensivos; Ambiente de instituciones de salud

\footnotetext{
${ }^{1}$ Especialista em Educação em Saúde e Mestranda em Enfermagem Fundamental pela Escola de Enfermagem de Ribeirão Preto - Universidade de São Paulo - USP - São Paulo (SP), Brasil.

${ }^{2}$ Especialista em Nefrologia e Educação em Saúde e Mestre em Enfermagem Fundamental pela Escola de Enfermagem de Ribeirão Preto - Universidade de São Paulo - USP - São Paulo (SP), Brasil.

3 Professora do Departamento de Enfermagem Psiquiátrica e Ciências Humanas da Escola de Enfermagem de Ribeirão Preto - Universidade de São Paulo - USP - São Paulo (SP), Brasil.
} 


\section{INTRODUÇÃO}

O estresse ocupacional, o qual se defronta o trabalhador moderno, se destaca como de especial importância, principalmente em algumas profissões como, por exemplo, a enfermagem. Embora seja fenômeno freqüentemente relacionado entre os enfermeiros para caracterizar a profissão, na prática há falta de instrumentos sistematizados para avaliar a problemática ${ }^{(1)}$.

A preocupação com o sofrimento e o prazer no trabalho dos profissionais de enfermagem surgiu com questionamentos relativos a forma como esses profissionais conseguiam suportar trabalho tão desgastante, principalmente pela necessidade de conviverem com o sofrimento, dor e a morte de modo tão freqüente ${ }^{(2)}$.

No estudo das reações do corpo às pressões exercidas pelas situações vivenciadas, surgiu o termo estresse, que é a soma das trocas inespecíficas do organismo em resposta a um estímulo ou situação. Foi descrita a chamada Síndrome Geral de Adaptação (SGA), que pode ser entendida como "o conjunto de todas as reações gerais do organismo que acompanham a exposição prolongada ao estressor", e compreende três fases: reação de alerta ou alarme (taquicardia, palidez, fadiga, insônia, falta de apetite, pressão no peito, estômago tenso), de resistência ou adaptativa (isolamento social, incapacidade de se desligar do trabalho, impotência para as atividades, peso nos ombros) e de exaustão (depressão) ${ }^{(3)}$.

O estresse ocupacional é um estado em que ocorre desgaste anormal do organismo humano e/ou diminuição da capacidade de trabalho, devido basicamente à incapacidade prolongada de o indivíduo tolerar, superar ou se adaptar às exigências de natureza psíquica existentes em seu ambiente de trabalho ou de vida ${ }^{(4)}$.

Segundo especialistas em psicopatologia do trabalho e da Organização Mundial de Saúde (OMS), as situações que causam ansiedade ao trabalhador, desencadeando o estresse, geram desgastes não só emocionais, como também físicos, com manifestações desagradáveis que podem, com o seu agravo, desencadear doenças ${ }^{(5)}$.

Diversos autores retratam a enfermagem como profissão estressante, devido à responsabilidade pela vida das pessoas e proximidade com os clientes em que o sofrimento é quase inevitável, exigindo dedicação no desempenho de suas funções, aumentando a probabilidade de ocorrência de desgastes físicos e psicológicos $^{(6-8)}$.

Considerando os ambientes críticos, como as Unidades de Terapia Intensiva (UTI), o estresse tem constituído fator de risco à qualidade de vida dos trabalhadores de enfermagem e, embora seja o local ideal para o atendimento a pacientes agudos graves recuperáveis, parece oferecer um dos ambientes mais agressivos, tensos e traumatizantes do hospital ${ }^{(9-11)}$.

Essas unidades, consideradas como ambientes fechados, com grande absorção tecnológica, foram comparadas aos estabelecimentos penitenciários, como um "sistema peculiar de vida interna", que configura condições propícias para a formação e manutenção de padrões culturais específicos ${ }^{(12)}$.

Percebe-se que a grande maioria dos profissionais da equipe de enfermagem sente prazer em cuidar de pacientes graves, porém vivencia angústias intensas pelo fato de terem que realizar grande número de procedimentos complexos. Além disso, têm que manipular inúmeros equipamentos e realizar todas as atividades com iniciativa, rapidez e livre de qualquer erro, pois isso poderia implicar na morte do paciente. Outro fator que possivelmente contribui para o desgaste dos profissionais é o próprio "clima" dessas unidades, pois o ritmo de trabalho é bastante intenso e a todo momento está presente a possibilidade de agravos e de morte ${ }^{(2)}$.

Enfermeiros, pela função que desempenham, assumem as atividades mais complexas e que envolvem maior risco para os pacientes, além de serem responsáveis pelas atividades desenvolvidas por toda a equipe de enfermagem. Dessa forma, incorporam alto nível de responsabilidade, na tentativa de ter o controle absoluto sobre o trabalho, o que muitas vezes os levam a exigir de si mesmos atitudes sobre-humanas ${ }^{(2)}$.

Por temor das conseqüências de um erro para si e para o paciente, internalizam excessivamente o controle sobre o trabalho. Esse mecanismo pode levá-los ao desenvolvimento de uma espécie de "prontidão paranóide", isto é, internalização de sentimentos persecutórios na ausência de um perseguidor concreto. Esse mecanismo é adotado inconscientemente por enfermeiros como forma de proteção frente à imprevisibilidade de suas conseqüências, uma vez que, no cotidiano, o controle absoluto sobre o trabalho é quase impossível, o grupo encontra-se freqüentemente ameaçado diante da possibilidade de erros. Enfim, esses profissionais, para evitarem a perda de controle, os sentimentos de culpa e a punição tornam-se vigilantes de si mesmos, controladores atentos aos resultados de seus atos, e experimentam o temor pelas conseqüências de uma atitude desatenta ${ }^{(8,13)}$.

Alguns autores ressaltam a complexidade existente nas UTI, revelando a importância de se rever as questões que permeiam o relacionamento interpessoal da equipe de enfermagem nesse contexto, tendo em vista os problemas emergentes de circunstâncias em que as peculiaridades do ambiente ocasionam aos seus profissionais, e também evidenciam o nível de ansiedade e tensão provocado, sobretudo, pela elevada responsabilidade que a enfermagem enfrenta em seu cotidiano profissional. Esse fato ocorre devido às conseqüências das variáveis que 
intervêm nesse processo tais como: ambiente extremamente seco, refrigerado, fechado, iluminação artificial, ruído interno contínuo e intermitente, interrelacionamento constante entre as mesmas pessoas da equipe durante o turno, bem como a exigência excessiva de segurança, respeito e responsabilidade para o paciente em sofrimento, dor e com morte iminente, para a garantia da qualidade da assistência ${ }^{(8,14)}$.

Em virtude da vivência como enfermeiras no contexto da terapia intensiva, as pesquisadoras sentiram a necessidade de identificar, entre os profissionais enfermeiros, o estresse. Assim, considera-se a importância da revelação precoce de alterações e fontes que causem prejuízo à saúde do trabalhador e que sejam passíveis de intervenção.

\section{OBJETIVOS}

Verificar a existência de estresse entre enfermeiros que atuam na assistência a pacientes críticos em uma Unidade de Cuidados Intensivos e a fase em que se encontram os profissionais comprometidos, assim como traçar o perfil desses profissionais quanto aos aspectos pessoais e profissionais.

\section{MÉTODOS}

O estudo foi realizado na Unidade de Cuidados Intensivos da Unidade de Emergência do Hospital das Clínicas da Faculdade de Medicina de Ribeirão Preto da Universidade de São Paulo (UE-HCFMRP-USP).

A equipe de enfermagem é formada por 14 enfermeiros e a carga horária desses profissionais varia entre 30 e 40 horas semanais, distribuída em turnos diurnos, noturnos e rotativos.

A amostra foi constituída por um grupo de 12 enfermeiros lotados no local e que consentiram em participar da pesquisa. As autoras da pesquisa, que trabalham na unidade, foram excluídas da amostra. Para a coleta de dados, utilizaram-se os seguintes instrumentos:

- dados gerais para caracterizar fatores sociodemográficos, incluindo as seguintes variáveis: sexo, idade, tempo de formação universitária, turno de trabalho e carga horária de trabalho diário;

Inventário de Sintomas de Stress para Adultos de Lipp (ISSL) que visa identificar de modo objetivo se o indivíduo possui sintomas de estresse e a fase em que se encontra ${ }^{(15)}$.

O ISSL foi validado em 1994 e tem sido utilizado em pesquisas e trabalhos clínicos na área do estresse ${ }^{(16)}$.

Para tornar claro o processo de desenvolvimento do estresse é necessário considerar que o quadro sintomatológico varia, dependendo da fase em que se encontra.
$\mathrm{Na}$ fase de alerta, considerada a fase positiva do estresse, o ser humano se mobiliza por meio da produção da adrenalina, a sobrevivência é preservada e uma sensação de plenitude é freqüentemente alcançada. $\mathrm{Na}$ segunda fase, a da resistência, a pessoa automaticamente tenta lidar com os seus estressores, de modo a manter a homeostase interna. Se os fatores estressantes persistirem em freqüência ou intensidade, há quebra da resistência da pessoa e ela passa à fase de quase exaustão. Nessa fase, o processo de adoecimento se inicia e os órgãos que possuem maior vulnerabilidade genética, ou adquirida, passam a mostrar sinais de deterioração. Se não há alívio para o estresse por meio da remoção dos estressores, ou pelo uso de estratégias de enfrentamento, ele atinge a sua fase final, a da exaustão, quando doenças graves podem ocorrer nos órgãos mais vulneráveis como enfarte, úlceras, psoríase, depressão e outros $^{(16)}$.

Contou-se com auxílio de uma psicóloga que participou da análise dos inventários de respostas dos participantes do estudo.

A coleta de dados ocorreu no período de 1 a 30 de junho de 2005, até se completarem as respostas dos 12 sujeitos constantes da amostra. Os dados gerais, juntamente com os inventários a serem preenchidos, foram entregues a cada um dos enfermeiros da amostra pelas pesquisadoras que, nesse momento, explicitaram os objetivos da pesquisa. Ao término do preenchimento, orientou-se cada um dos sujeitos que colocasse o protocolo de respostas anonimamente em uma pasta guardada dentro de um armário, previamente designado, para que as autoras o recolhesse num prazo estabelecido de sete dias.

Antes do início da coleta de dados, o presente estudo obteve a aprovação da Comissão de Ética em Pesquisa do referido hospital; os sujeitos da pesquisa foram informados quanto aos objetivos, orientados quanto ao sigilo de sua identificação e de suas respostas, assinando um Termo de Consentimento Livre e Esclarecido.

\section{RESULTADOS}

Atendendo aos objetivos do estudo, traçou-se um perfil sociodemográfico da amostra em busca de aspectos que pudessem estar relacionados ao desenvolvimento do estresse.

A Tabela 1 mostra que quase a totalidade dos enfermeiros $(91,7 \%)$ era do sexo feminino.

Esse resultado é convergente com outros trabalhos que argumentam ser a enfermagem, desde seu início no século XIX, considerada como essencialmente feminina. Há autores, ainda, que reforçam que o cuidar em enfermagem é uma extensão das atividades realizadas na manutenção da família ${ }^{(1,7,17)}$. 
Tabela 1 - Distribuição dos enfermeiros lotados na Unidade de Cuidados Intensivos do HCFMRP-USP (UE), segundo o sexo e a faixa etária

\begin{tabular}{lrr}
\hline Variáveis & $\mathbf{n}^{\mathbf{0}}$ & \multicolumn{1}{c}{$\mathbf{0}$} \\
\hline Sexo & 11 & 91,7 \\
Feminino & 1 & 8,3 \\
Masculino & $\mathbf{1 2}$ & $\mathbf{1 0 0}$ \\
\hline Total & & \\
Faixa Etária (anos) & & \\
\hline$<20$ & 4 & 33,3 \\
$20-30$ & 3 & 25,0 \\
$30-40$ & 5 & 41,7 \\
$40-50$ & 0 & 0 \\
Menor $=50$ & $\mathbf{1 2}$ & $\mathbf{1 0 0}$ \\
\hline Total & & \\
\hline
\end{tabular}

Considerando que a mulher pode suportar cerca da metade da carga física que o homem suporta, esse resultado figura como fator relevante no trabalho de enfermagem, já que nas unidades de cuidados intensivos, a demanda de esforço físico é grande, devido ao peso dos pacientes acamados e que, muitas vezes, se encontram impossibilitados de auxiliarem em qualquer tipo de movimentação $\mathrm{O}^{(1,18)}$.

Estudiosos ressaltam que o dispêndio elevado de força muscular e gasto excessivo de energia física têm ocasionado problemas posturais e fadiga geral nos trabalhadores, tornando-se mais grave na medida em que se constata predominância de mulheres na força de trabalho empregada no hospital ${ }^{(19)}$.

Quanto à faixa etária, conforme a Tabela 1 , a maioria da amostra estudada $(41,7 \%)$ encontrava-se entre 40 e 50 anos de idade, portanto, pessoas mais maduras e, possivelmente, com experiências anteriores de inserção no mercado de trabalho, achados esses que estão em consonância com os dados da literatura ${ }^{(1,17)}$.

Nesse contexto, segundo alguns autores, a exposição prolongada e contínua a estressores no ambiente de trabalho pode desencadear exaustão física e psíquica, além de determinar um processo insidioso de estresse. Além disso, as atividades em terapia intensiva exigem elevado grau de agilidade, destreza física e energia, características essas mais comuns em indivíduos jovens $^{(17,20)}$.

Em relação ao tempo de formação universitária (Tabela 2), foi predominante o período entre 5 e 10 anos (33,4\%). A instituição admite profissionais sem experiência, e esses geralmente são treinados no próprio serviço, o que poderia explicar a maior porcentagem com pouco tempo de habilitação. Além disso, pode-se sugerir que, como a maior parte é formada por pessoas de idade mais elevada, a inserção no mercado de trabalho ocorreu tardiamente, talvez pelas dificuldades financeiras que atualmente se encontram para concluir o ensino superior.
Tabela 2 - Distribuição dos enfermeiros lotados na Unidade de Cuidados Intensivos do HCFMRP-USP (UE), segundo tempo de formação universitária, turno de trabalho e carga horária

\begin{tabular}{lrr}
\hline $\begin{array}{l}\text { Variáveis } \\
\text { Tempo de Formação }\end{array}$ & $\mathbf{n}^{\mathbf{0}}$ & $\mathbf{\%}$ \\
\hline$<3$ anos & 3 & 25,0 \\
3 - 05 anos & 3 & 25,0 \\
$5-10$ anos & 4 & 33,4 \\
10 - 15 anos & 1 & 8,3 \\
$15-20$ anos & 1 & 8,3 \\
\hline Total & 12 & 100,0 \\
\hline Turno de Trabalho & & \\
\hline Diurno & 5 & 41,7 \\
Noturno & 4 & 33,3 \\
Rodízio & 3 & 25,0 \\
\hline Total & 12 & 100,0 \\
\hline Carga Horária de Trabalho & & \\
\hline 6 horas/diárias & 3 & 25 \\
10 -12 horas/diárias & 9 & 75
\end{tabular}

No estudo, $5(41,7 \%)$ dos sujeitos exerciam suas atividades no período diurno, seguidos de $4(33,3 \%)$ no noturno e $3(25 \%)$ em turnos rotativos, de acordo com a Tabela 2. Isso, em decorrência das necessidades institucionais de escalas de trabalho, tendo em vista a demanda do serviço para o atendimento.

$\mathrm{E}$, apesar de pequena porcentagem exercer suas atividades em turnos rotativos, pode-se ressaltar que se torna fator de agravo ao estresse, isto é, pode afetar a eficiência do trabalhador, sua saúde física e psicológica, seu bem-estar, sua família e vida social, pelo fato de o estresse modificar os ritmos biológicos, levando à dificuldade e lentidão de sincronização desses ritmos, devido às modificações do ciclo sono-vigília, que induzem ao estado de desgaste nesse trabalhador ${ }^{(21)}$.

Verificou-se que mais da metade da amostra, 9 (75\%) trabalhava entre 10 e 12 horas diárias, resultando em carga horária exaustiva, considerando o tipo de trabalho e as atribuições que exercem (Tabela 2). Por se tratar de unidade onde se tornam freqüentes as situações de urgência, com ritmo de trabalho intenso, desgastante e com a presença constante de patologias graves, a dor e o sofrimento do paciente hospitalizado em terapia intensiva podem sobrecarregar emocional e afetivamente o profissional que atua por longos períodos nessa unidade.

A duração da atividade, hora, ou execução da tarefa, e pressão dos ritmos e de horários do meio social são elementos decisivos da dificuldade laboral, sendo importantes fatores de processos fisiológicos e psicológicos para o trabalho ${ }^{(22)}$.

O instrumento para avaliação de sintomas de estresse identificou que $8(66,7 \%)$ enfermeiros da amostra apresentavam estresse, isto é, número elevado de sintomas físicos e psicológicos que os classificam como estressados, supondo que há ocorrência de desgaste de energia maior do que a reposição e o organismo 
encontra-se em desequilíbrio. Os demais sujeitos encontravam-se sem estresse, ou estavam conseguindo repor suas energias.

O sujeito é capaz de enfrentar o estresse quando utiliza esforços cognitivos e comportamentos definidos como mecanismos de "coping", para identificar, administrar, avaliar e manter o equilíbrio em resposta ao estressor ${ }^{(23)}$.

Portanto, se para $4(33,3 \%)$ desses indivíduos não houve estresse, isso indica que houve resposta de adaptação, equilíbrio homeostático e a utilização de estratégias de coping eficientes. Todos os enfermeiros encontravam-se na fase de resistência, não sendo evidenciado em nenhum profissional a fase de quaseexaustão ou exaustão.

A fase de resistência aparece se houver a persistência do estressor e a compatível adaptação e, nessa fase, os sinais de reação de alarme virtualmente desaparecem. Já a exaustão ocorre na constância do estressor e quando os mecanismos de enfrentamento não são suficientes ${ }^{(3)}$.

\section{DISCUSSÃO}

No presente trabalho buscou-se caracterizar um grupo de enfermeiros atuantes em terapia intensiva de um hospital de grande porte do interior paulista e avaliar os sintomas de estresse. A maior parte do grupo foi composta pelo sexo feminino, o tempo de formação variou até 20 anos, a faixa etária de 20 a 50 anos, e a carga horária de trabalho entre 10 e 12 horas por dia.

O ambiente de trabalho, como se constatou, é consideravelmente estressante; as atividades desenvolvidas exigem alto grau de responsabilidade e qualificação, com desgaste emocional intenso. É possível que, por a unidade intensiva estar localizada num serviço de atendimento emergencial, a tensão se mostre tão aumentada.

A avaliação do estresse por meio de um instrumento que focaliza as reações do indivíduo sejam elas psicológicas, fisiológicas ou comportamentais, mostrou que a maioria apresentava sintomas indicativos de sobrecarga.

Ser enfermeiro em serviços de terapia intensiva e estar na fase de resistência pode significar diminuir o estado de alerta em relação a um paciente crítico que necessita de vigilância constante ${ }^{(24)}$.

Em outro estudo sobre a temática, utilizou-se metodologia distinta - a avaliação do trabalho em si como indicador de sua potencialidade estressora, além da focalização de aspectos da saúde mental dos enfermeiros e estratégias de enfrentamento, mobilizadas em difíceis situações laborais. Diferentemente dos resultados do presente estudo não se identificaram avaliações, para a maioria dos participantes, que qualificassem o trabalho como excessivamente estressor e, ainda não havia comprometimentos na avaliação da própria saúde mental. Em relação a essa última consideração, deve-se salientar que, embora os instrumentos sejam bastante diferentes, avaliam aspectos semelhantes como estresse psíquico e sintomas psicossomáticos, entre outros. Identificou-se mecanismos adaptativos de enfrentamento que possivelmente auxiliavam na manutenção da integridade dos profissionais ${ }^{(25)}$.

Chama a atenção no presente estudo, a carga horária das atividades dos profissionais que sugere acúmulo de empregos, ao considerar que a jornada diária é de seis horas, excetuando-se plantões. Diante disso, pode-se pensar que os resultados verificados quanto ao estresse devem-se a essa característica da maioria dos participantes deste estudo e não apenas à atuação em terapia intensiva. Essa é uma indagação que merece ser abordada por outros trabalhos e que, no presente, limita as conclusões sobre essa pequena amostra aqui focalizada.

Não foi objeto de investigação a verificação de ocorrências de adoecimento que requeressem afastamento das atividades laborais; ou, ainda, registro de situações conflituosas no cotidiano de trabalho que expusessem os enfermeiros a tensões e pudessem mostrar, sob outra perspectiva, descrição das atividades dos indivíduos focalizados. Entretanto, acredita-se que essa abordagem ampliaria a compreensão da atuação do enfermeiro em terapia intensiva.

A partir de estratégias metodológicas semelhantes a outros estudos, verificou-se resultados coincidentes em relação à presença de estresse no enfermeiro que atua em terapia intensiva. Portanto, é de suma importância para a saúde física e mental desses profissionais que eles saibam identificar as manifestações do processo de estresse, e que aprendam a detectar quais são os estressores que desencadeiam o processo, pois, dessa forma, poderão utilizar "mecanismos de enfrentamento" eficientes para a adaptação ao estressor e, conseqüentemente, interromper a evolução do processo de estresse $e^{(1,7,17,25)}$.

Ainda que limitado aos achados num contexto específico, os dados encontrados sugerem que há direcionamento de atenção aos profissionais, quer no sentido de minimizar a sobrecarga, quer no sentido de evitar agravamentos.

Diante do exposto, torna-se prudente investir maiores esforços na prevenção do estresse como controle da saúde dos trabalhadores de enfermagem, proporcionando maior rendimento no trabalho, ambiente agradável, seguro e assistência de qualidade ${ }^{(25)}$.

\section{CONCLUSÃO}

Neste estudo, que abordou enfermeiras num centro de terapia intensiva quanto à identificação de sintomas de estresse, verificou-se que a maior parte delas mostrou estar vivenciando sintomas físicos e psicológicos. Embora 
não se pudesse analisar estatísticamente a associação de tais sintomas com características sócio demográficas deste grupo de 12 enfermeiros, chamou atenção a jornada diária de trabalho da maior parte deles.

A sistematização dos dados desta pequena amostra traz indicativos de uma associação de estresse com duração da jornada que merece ser explorada em outros trabalhos e que, para este grupo específico, sugere uma realidade que pode ser analisada com vistas à redução dos sintomas apresentados.

\section{REFERÊNCIAS}

1. Meirelles NF, Zeitoune RCG. Satisfação no trabalho e fatores de estresse da equipe de enfermagem de um centro cirúrgico oncológico. Esc Anna Nery Rev Enferm. 2003; 7(1): 78-88.

2. Shimizu HE, Ciampone MHT. Sofrimento e prazer no trabalho vivenciado pelas enfermeiras que trabalham em Unidades de Terapia Intensiva em um hospital escola. Rev Esc Enfermagem USP. 1999; 33(1): 95-106.

3. Selye H. The stress of life. New York: McGraw Hill; 1956.

4. Couto HA. Stress e qualidade de vida dos executivos. Rio de Janeiro: COP; 1987.

5. Organização Mundial de Saúde. Identificación de enfermedades relacionadas con el trabajo y medidas para combartirlas. Genebra: Organização Mundial de Saúde; 1985. [Série de Informes Técnicos, 714].

6. Bianchi ERF. Stress entre enfermeiros hospitalares [tese]. São Paulo: Universidade de São Paulo; 1999.

7. Miquelim JDL, Carvalho CBO, Gir E, Pelá NTR. Estresse nos profissionais de enfermagem que atuam em uma unidade de pacientes portadores de HIV - AIDS. DST J Bras Doenças Sex Transm. 2004; 16(3): 24-31.

8. Hoga LAK. Causas de estresse e mecanismos de produção do bem-estar dos profissionais de enfermagem de unidade neonatal. Acta Paul Enfermagem. 2002; 15(2): 18-25.

9. Holland C, Cason CL, Prater LR. Patients' recollections of critical care. Dimens Crit Care Nurs. 1997; 16(3): 132-41.

10. Russel S. An exploratory study of patients' perceptions, memories and experiences of an intensive care unit. J Adv Nurs. 1999; 29(4): 783-91.

11. 11. Bianchi ERF. Estresse em enfermagem: análise da atuação do enfermeiro de centro cirúrgico [tese]. São Paulo: Universidade de São Paulo; 1990.

12. Fischer RM. O círculo do poder: as práticas invisíveis de sujeição nas organizações complexas. In: Fleury MTL, Fischer RM, coordenadores. Cultura e poder nas organizações. São Paulo: Atlas; 1989. p. 65-88.

13. Borsoi ICF. Saúde mental e trabalho: um estudo de caso da enfermagem [tese]. São Paulo: Pontifícia Universidade Católica; 1992.

14. Pereira MER, Bueno SMV. Lazer: um caminho para aliviar as tensões no ambiente de trabalho em UTI: uma concepção da equipe de enfermagem. Rev Latinoam Enfermagem. 1997; 5(4): 75-83.

15. Lipp MN. Manual do inventário de sintomas de stress para adultos de Lipp (ISSL). São Paulo: Casa do Psicólogo; 2000.

16. Lipp MN, Guevara AJH. Validação empírica do inventário de sintomas de stress. Est Psicol. 1994; 11(3): 43-9.

17. Costa JRA, Lima JV, Almeida PC. Stress no trabalho do enfermeiro. Rev Esc Enfermagem USP. 2003; 37(3): 63-71.

18. Waldow VR. Cuidar / cuidado: o domínio unificador da enfermagem. In: Waldow VR, Lopes MJM, Meyer DE. Maneiras de cuidar, maneiras de ensinar: a enfermagem entre a escola e a prática profissional. Porto Alegre: Artes Médicas; 1995. p. 7-30.

19. Guedes EM, Mauro MYC. (Re)visando os fatores de risco e as condições de trabalho da enfermagem hospitalar. Rev Enfermagem UERJ. 2001; 9(2): 144-51.

20. Miranda AF. Estresse ocupacional: inimigo invisível do enfermeiro? [tese]. Ribeirão Preto: Escola de Enfermagem de Ribeirão Preto. Universidade de São Paulo; 1998.

21. Fisher FM, Lieber RR, Brown FM. Trabalho em turnos e relação com saúde-doença. In: Mendes R. Patologia do trabalho. São Paulo: Atheneu; 1996. p. 545-72.

22. Bulhões I. Riscos do trabalho de enfermagem. 2a ed. Rio de Janeiro: Ideas; 1998.

23. Lazarus RS. Stress and emotion: a new synthesis. New York: Springer Publishing Co.; c1999.

24. Hay D, Oken D. The psychological stresses of intensive care unit nursing. Psychosom Med. 1972; 34(2): 109-18.

25. Da Silva Britto E, Pimenta Carvalho AM. Stress, coping (enfrentamento) e saúde geral dos enfermeiros que atuam em unidades de terapia intensiva e problemas renais. Enfermería Global [periódico na Internet]. 2004 [citado 2005 Jun 12]; 4: [cerca de 14 p.]. Disponível em: www.um.es/eglobal 